health status of farmers who ahve been using pesticides for the past years. Laboratory examination of blood was also done, including blood cholinesterase to dertermine organophosphate exposures.

Results Majority were males (53.4\%), married (80.5\%) with a mean age of 47 years old. The most commonly used pesticides were Tamaron (36.1\%), Dithane (34.1\%), Sumicidine (29.0\%), Selecron (24.9\%) and Lannate (15.2\%). Tamaron, being the most commonly used, has an active ingredient of methamidophos and classified as an organophosphate pesticide. Meanwhile, Dithane, a mancozeb, is a dithiocarbamate pesticide and Sumicidine is a pyrethroid with an active ingredient of fenvalerate. In addition, Selecron is composed mainly of ingredients of prochloroz $\mathrm{mn}$ and spinosad, respectively (Table 2). During their agricultural work, farmers used pesticides mainly for three to six hours per day $(51.2 \%)$, one to two days weekly $(86.8 \%)$ and three to four weeks per month (82.3\%). 40.9\% who underwent the physical examination were diagnosed to have abnormal assessment results. Analyses indicated that pesticide use and risk factors were found to have association at $p=0.05$ with easy fatigability, weight loss, loss of appetite, cerebellar function, creatinine levels, haemoglobin, mean corpuscular volume, mean corpuscular haemoglobin count, and platelet count. 5.3\% of the farmers were found to have abnormal platelet count. Platelet count may indicate whether the patient is having bleeding problems, infectious processes, bone marrow depression, malignancies or anaemia. For RBC cholinesterase activity, it was found that $50.8 \%$ of the samples showed depression in activity.

Conclusions This study has demonstrated the interaction between the farmers' pesticide exposure and work practices, as well as physical, neurological and laboratory assessment findings. The study shows that farmers are at a considerable risk of either acute or chronic pesticide poisoning because of improper work practices. Around $40.9 \%$ of the farmers were diagnosed to have abnormal physical examination findings while $4.6 \%$ presented problems in their neurological functioning and less than $10 \%$ of the farmers exhibited abnormal laboratory results. The author recommends the use of the information gathered in this study to improve current policies and standards with regards to surveillance of pesticide use.

\section{TRENDS OF OCCUPATIONAL INJURY IN THE PHILIPPINES: IMPLICATIONS FOR POLICY}

Jinky Leilanie Lu. National Institutes of Health, Univeristy of the Philippines Manila, Manila, The Philippines

\subsection{6/oemed-2014-102362.176}

Objectives This study aimed to review and assess the prevalence and incidence of occupational injuries in the Philippines.

Method Data collection were done from various agencies, namely, Bureau of Labour and Employment Statistics (BLES) of the Department of Labour and Employment (DOLE), Labour Force Survey of National Statistics Office, among others. Hospital-based surveys and newspaper reports were also sources of data for this study.

Results The review showed that about 358000 fatal and 337 million non-fatal occupational accidents in the world, and 1.95 million deaths from work-related diseases. Occupational injuries in the Philippines showed 22265 cases in 2003, and 47235 cases in 2007. The manufacturing industries registered the highest number of cases Out of the reported cases of occupational injuries, 178 resulted in death in 2000, and 116 deaths in 2007. Injury occurred at 6 injury cases per 500 full-time workers, or 1 injury case for every 88 workers in 2000. In the following years, it declined to 4 cases per full-time worker in 2003, and 3 cases for every 88 workers in 2007. Based on hospital records, there was a total of 9521 injury cases reported for the first quarter of 2010 in 77 government and private hospitals in the country. The cause of injury mostly occurred on the road (44.4\%), and workrelated injuries were reported at $7.8 \%$.

Conclusions The review of occupational injuries in the Philippines showed major trends in injuries, causes of injuries and rates and severity of injury. However, the data lack more specific and segregated information per industry and occupational grouping, as well as identification of risk factors associated with these injuries. Therefore, improvements in injury surveillance and documentation of injury cases as well as research into risk factors at work should be done. All these efforts should lend towards prevention strategies and guidelines on occupational injuries in the Philippines. Also, there is a need to have a standard nomenclature of occupational injuries, starting from the primary data sources (company clinics) which are the bases of the national data. It is suggested that data collection on occupational injuries be a national scale, and not merely randomised collection of data for small, medium and large industries.

\section{A NEW MODEL FOR OUTREACH TO SMALL INDUSTRIAL FIRMS: SAFETY AUDIT RESULTS FROM THE NATIONAL MACHINE GUARDING PROGRAM}

${ }^{1}$ Samuel Yamin, ${ }^{1}$ David Parker, ${ }^{2}$ Lisa Brosseau, ${ }^{1}$ Min Xi, ${ }^{3}$ Bob Gordon. ${ }^{1}$ Park Nicollet Institute, St. Louis Park, MN, USA; ${ }^{2}$ University of Illinois at Chicago, Chicago, IL, USA; ${ }^{3}$ Interrobang Group, Minneapolis, MN, USA

\subsection{6/oemed-2014-102362.177}

Objectives Small industrial firms frequently lack occupational safety and health (OSH) expertise. In addition, development of effective, widely applicable OSH interventions for small businesses has proven to be a persistent challenge. The National Machine Guarding Program is an intervention currently underway to address these issues among small (3-150 employees) metal fabrication businesses.

Method A partnership was formed with workers' compensation insurers to recruit businesses for an intervention designed to prevent machine-related injuries and improve safety programs such as lockout/tagout. Participants receive a baseline evaluation, two intervention visits, and a follow-up evaluation. Pooled results from baseline safety assessments will be presented.

Results 221 businesses from 29 U.S. states were enrolled. At baseline, the mean score for machine safety audit was $73.7 \%$, however, only $67.5 \%$ of items concerning point of operation safeguards were present on average. For shop-wide safety programs and policies, mean audit score was $43.4 \%$. Safety program/policy scores were significantly lower among smaller businesses, whereas machine safeguarding equipment scores were similar across all size strata.

Conclusions The National Machine Guarding Program represents a new intervention model for widespread outreach to small industrial firms. Baseline safety audit results show a need for improvement in shop-wide safety programs and in critical areas of machine safeguarding. Preliminary results indicate that this is an effective model for recruiting small metal fabrication businesses into a nationwide intervention. Effectiveness of intervention programs in improving these aspects of injury prevention will be evaluated to determine whether broader application is warranted. 